Does the random distribution of discrete photoreceptor events limit the sensitivity of the retina?

\title{
Reuter, T.
}

Elsevier

1986

Neuroscience Research Supplement. 1986. 4: S163-S180

http://hdl.handle.net/1975/944

Downloaded from Helda, University of Helsinki institutional repository.

This is an electronic reprint of the original article.

This reprint may differ from the original in pagination and typographic detail.

Please cite the original version. 
DOES THE RANDOM DISTRIBUTION OF DISCRETE PHOTORECEPTOR EVENTS LIMIT THE SENSITIVITY OF THE RETINA?

TOM REUTER*, KRISTIAN DONNER * and DAVID R. COPENHAGEN* *

*Department of zoology, University of Helsinki, SF 00100 Helsinki, Finland.

* Department of Ophthalmology and Physiology, University of California School of Medicine, San Francisco, CA. 94143, U.S.A.

\section{INTRODUCTION}

In this study we investigate to what extent the sensitivity and reliability of the signal transmission through the retina can be understood on the basis of simple statistical signal-to-noise considerations. The eyes of anuran amphibians (frogs and toads) are well suited for a study of this kind: (1) in their natural environment toads (Bufo bufo) often detect and catch their prey at an ambient illumination producing only $0.1-1$ isomerizations per rod and secondlcalculations based on data presented by Larsen and Pedersen (28), with details kindly provided by Dr. Lis Olesen Larsen). As will become clear below, it is thus probable that the reliability of their scotopic vision is strongly influenced by photon noise; (2) it is possible to intracellularly record light responses from all the main classes of neurones in the amphibian retina; (3) it is well known how toad rods respond to the photoisomerization of single rhodopsin molecules (14), and we also know which is of crucial importance for a signal-to-noise analysis that in complete darkness these rods produce spontaneous noise events kinetically indiscernible from those produced by photoisomerizations (15). These dark events are randomly distributed in timewithin each outer segment. In a single Bufo marinus rod at $20^{\circ} \mathrm{C}$ the dark events occur with a mean frequency of $1-2$ events per minute.

We first report results obtained in intracellular recordings from toad rods and horizontal cells. We then analyse extracellularly measured responses of frog ganglion cells to short flashes of light presented in darkness or against weak backgrounds. The upper limit of the signal-to-noise ratios (SNR) of the response of a retinal cell under these conditions is given by the equation

$$
S N R=E_{S} / \sqrt{E_{S}+E_{B}+E_{D}}
$$

where $E_{S}$ is the mean number of isomerizations (within the receptive field of the cell) produced by the flash stimulus, EB is tho mean 
number of background-induced isomerizations interacting with the stimulus, $\left(E_{B}=0\right.$ for darkness) and $E_{D}$ the mean number of dark isomerization-like events interacting with the stimulus $(10)$.

The number of background-elicited events and dark events interacting with the stimulus is computed when one knows the mean frequency of these events, the size of the receptive field of the cell and the integration time $\left(\underline{t}_{i}\right)$ of the response. If the linear response to a weak $\mathrm{flash}$ is $\mathrm{f}(\underline{t})$ normalized to $\mathrm{f}(\underline{t})=1$ at the peak of the response, the integration time is defined as $\int_{0}^{\infty} f(\underline{t}) d t$. From this definition it follows that the step sensitivity, $\left.\mathrm{S}_{\mathrm{S}}(\mathrm{mV} \times \mathrm{s} / \mathrm{Rh})^{*}\right)$, is equal to the flash sensitivity, $\mathrm{S}_{\mathrm{F}}\left(\mathrm{mV} / \mathrm{Rh}^{*}\right)$, multiplied by $\underline{t}_{i}(13) ; \mathrm{Rh}$ * signifies one photoisomerization per rod. In extracellular ganglion cell recordings we define sensitivity as reciprocal of $\mathrm{flash}$ intensity $\left(1 / \mathrm{Rh}^{\star}\right)$ at threshold.

Rod mediated vision exhibits a very high sensitivity. However, high sensitivity alone does not guarantee good vision in dim light. High reliability, i.e. a high SNR, is equally important. By definition, high sensitivity means that a small number of isomerizations is required at threshold, while high reliability means that flash intensities below and above the threshold have low and high probabilities, respectively, of producing responses. For very dim lights, there is a large variation in the number of photons striking the retina with each flash. This variability follows a Poisson distribution and can be considered as photon noise. Additional noise sources (dark rod events, synaptic noise in the retina, etc.) combine with photon noise to degrade the reliability (SNR) of ganglion cells. Ultimately the noise will limit sensitivity too, since ever increasing stimulus intensities are required to exceed the noise.

We may study the mechanism of the signal-to-noise discrimination of a cell at three levels: (1) The maximum possible SNR of a response to a given flash can be calculated. This can be done by applying Eq. (1) after determining the receptive field size, integration time, the rate of dark events and the intensity of the background; (2) The physiologically realized SNR of a given cell can be obtained if the fluctuations in membrane potential due to stimuli and the fluctuations due to noise are recorded intracellularly. For spiking cells, a SNR can be obtained from extracellular spike recoldings which are long and stable enough for the determination of frequency of response functions; (3) Finally 
the origins of an observed SNR can be analysed by a study of the synaptic mechanisms and membrane properties determining the sensitivity and SNR of a cell.

The questions asked in this paper mainly refer to the first two of the three levels. By relating the SNR obtained at level (2), the physiological SNR, to the SNR calculated at level (1), the maximum possible SNR, we can see how closely the simple SNR considerations expressed by Equation (1) describe the neuronal processing of information in the retina.

\section{METHODS AND BIOLOGICAL DATA}

In the San Francisco laboratory, intracellular microelectrode recordings were made in dark-adapted eyecup preparations of the toad, Bufo marinus (temp. $20^{\circ} \mathrm{C}$ ). The dissection and maintenance of the eye, and the light stimulation and recording system are described elsewhere $(17,18,19)$. Microelectrodes with resistances of 500-900 M $\Omega$ were fabricated on a Brown-Flaming air blast puller. Membrane potentials were amplified, filtered and recorded on magnetic tape for later analysis. The identity of representative cells was confirmed by intracellular Lucifer Yellow staining (2).

In the Helsinki laboratory, extracellular ganglion cell recordings were made with glass micropipettes ( $3 \mathrm{M} \mathrm{NaCl)}$, resistance about $20 \mathrm{M} \Omega$ ) in dark-adapted eyecups of the common frog, Rana temporaria (temp. $11.5^{\circ} \mathrm{C}$ ). The optics, the recording system and the display of the responses are described elsewhere $(6,21,22)$. Our criteria for identifying different ganglion cell classes are discussed by Bäckström and Reuter (7). All ganglion cell data presented in this study refer to the oN-response or flash response of class $1-2$ or class 3 units.

The ganglion cell threshold was estimated by collecting a sufficient number of "positive" and "negative" trials at $0.1-0.4$ log unit intensity intervals; in practice ten to twenty flashes at $45 \mathrm{~s}$ intervals were used for determining the threshold, i.e. the intensity having a 508 probability of eliciting a spike response within a period of $2.5 \mathrm{~s}$ following the flash.

The integration time of extracellularly recorded ganglion cell responses were obtained by the equation $\underline{t}_{i}=I_{F} / I_{S}$ where $I_{F}(R h *)$ and ${ }^{I} S\left(R{ }^{*} S^{-1}\right)$ are the flash and step threshold intensities, respectively. The receptive field area of a ganglion cell was measured by determining area-threshold functions (for the validity of the method, see 
ref. 21), while the receptive fields of horizontal cells were measured with slit stimuli and by determining stimulus area response amplitude functions (29). The data from a given cell were fitted to a two-dimensional Gaussian distribution with a certain standard deviation, $\sigma$. The value $\sigma \sqrt{2}$ was used as receptive field radius (19).

Except for the temperature, the treatment of the toad and frog eyecups was very similar in San Francisco and Helsinki. In both laboratories the light reached the eye through two channels, one for steady background illumination, the other for step or flash stimulation. All flash stimuli $(13$ or $67 \mathrm{~ms})$ used were much shorter than the integration times of the cells. $500 \mathrm{~nm}$ or $512 \mathrm{~nm} 1$ ights were used for both stimuli and backgrounds. Extreme care was taken to prevent stray light from entering the Faraday cage containing the specimen chamber. The light intensities were measured with several separately calibrated radiometers, and in Helsinki the intensity was further calibrated against a rhodopsin extract.

The effective collecting area of a toad rod for $500 \mathrm{~nm}$ light was taken as $29.5 \mathrm{~mm}^{2}$ (24). The light absorption by the red rods in the frog retina was spectrophotometrically determined (27). Our own microscopic investigations showed that the mean densities of red rods were $15000 / \mathrm{mm}^{2}$ and $15700 / \mathrm{mm}^{2}$ in the central retinae of Bufo and Rana, respectively, and we have estimated that in these species 448 and 368 of the incident $500 \mathrm{~nm}$ photons produce photoisomerizations in red rods (less than 38 is absorbed in other photoreceptors).

In recordings from the distal $3 / 4$ of the Bufo marinus rod outer segment (ROS) Baylor et al. (15) determined the frequency of dark isomerization-like events to be $0.021 \mathrm{Rh}^{*} \mathrm{D}^{-1}$ at $20^{\circ} \mathrm{C}$ which corresponds to $0.028 \mathrm{Rh}{ }^{\star} \mathrm{D}^{-1}$ in the complete rod. When corrected for temperature and rod size the frequencies of dark rod events $\left(R h{ }^{*} D^{S^{-1}}\right.$ ) are very similar in dogfish, toad and monkey $(3,15,16)$, and thus it is reasonable to assume that the same applies to the two anurans Bufo marinus and Rana temporaria. After correcting for temperature (ref. 15, Fig. 9) and for the fact that the mean length of Rana ROSs is only 80 of of the length of Bufo ROSs, we estimate the rate of dark events in Rana rods at $11.5^{\circ} \mathrm{C}$ to $0.006 \mathrm{Rh}^{\star} \mathrm{D}^{\mathrm{S}^{-1}}$.

Although this paper does not describe extracellular recordings from toad ganglion cells, we have carried out such experiments both in San Francisco and in Helsinki (19). In San Francisco, 
the mean threshold of fully dark-adapted Bufo ganglion cells (large spot stimuli) was found to be $0.028 \mathrm{Rh} *(n=10)$, while it was $0.031 \mathrm{Rh} *(n=9)$ in Helsinki. Thus we think that the stimulation and recording conditions were similar in San Francisco and Helsinki.

\section{RESULTS}

Qualitative aspects of intracellularly recorded photon noise

Fig. I shows intracellular responses of a rod, horizontal cell

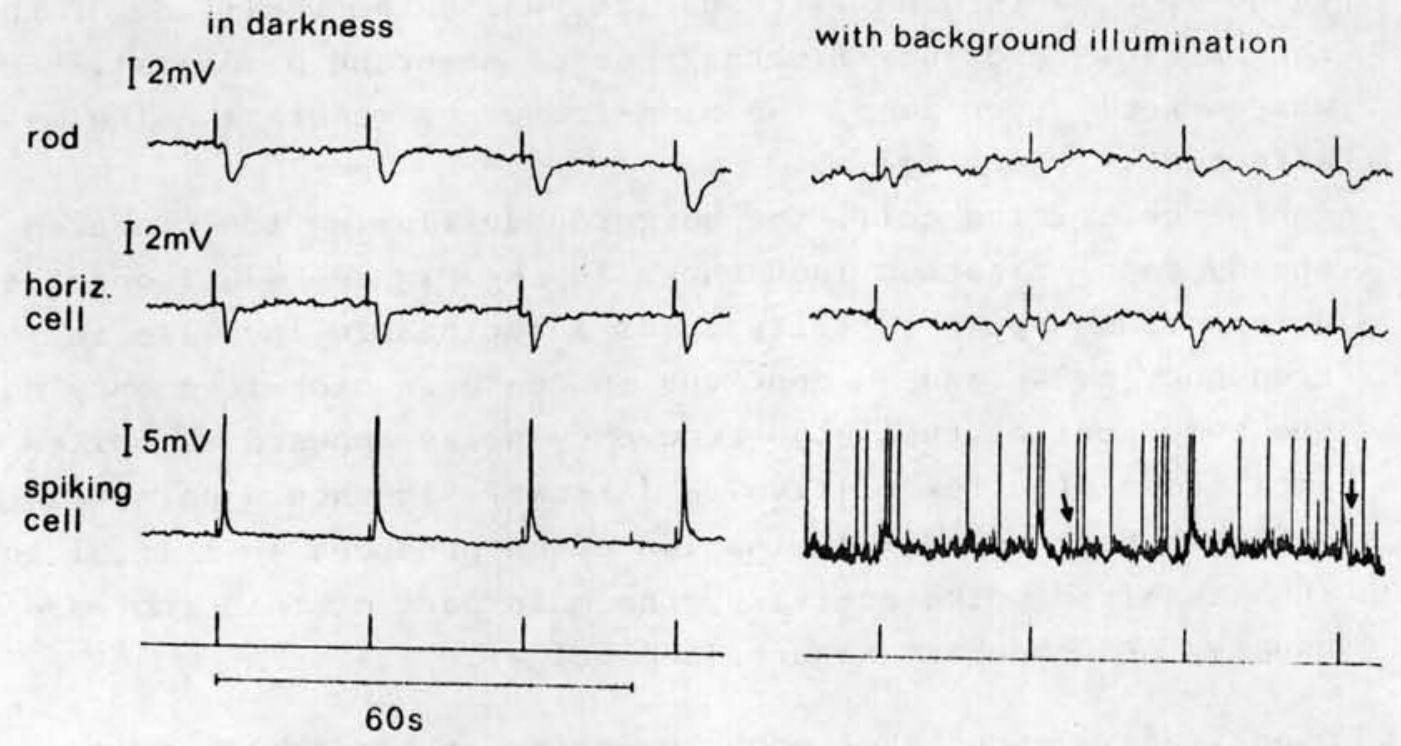

Fig. 1. Intracellular recordings from a rod, a horizontal cell and a spiking cell (probably a ganglion cell, but as it was not stained we cannot exclude the possibility that it was an amacrine ce11). The spikes are truncated and only about 408 of their amplitude is shown. In the right half the cells were exposed to full field backgrounds, $1.83 \mathrm{Rh}^{*} \mathrm{~s}^{-1}$ for the rod and spiking cell and $0.92 \mathrm{Rh}^{*} \mathrm{~s}^{-1}$ for the horizontal cell. These backgrounds caused -1.5 and $-10 \mathrm{mV}$ steady hyperpolarizations in the rod and horizontal cell, respectively, and a $4 \mathrm{mV}$ depolarization in the spiking cell. Flashes with $0.35 \mathrm{~mm}$ spot stimuli were given at $22 \mathrm{~s}$ intervals as indicated under the recordings, $2 \mathrm{Rh}^{\star}$ for the rod and $0.3 \mathrm{Rh}$ * for the horizontal cell and spiking cell. The upward "spikes" seen in the recordings and just preceding the flashes are pulses for amplitude calibration. Membrane potential lecoraings from single rods do not show discrete photoismerization events. The reason for this is that each rod is electrically connected to many other rods which dilute and average polentid signals from individual photoisomerizations $(17,23,29,32)$. In the Bufo malinus retina, a group of 36 rods can be considered to represent the receptive field of a single rod. The corresponding numbers for typical horizontal cells and ganglion cells are 250 and 2000 , respectively (19). 
and ganglion cell in the Bufo retina. The responses to a series of identical flashes are shown in darkness and during background illumination. These records show how the flux of randomly distributed photons in a relatively dim background light (1-2 $\mathrm{Rh}^{\star} \mathrm{S}^{-1}$ ) degraded the SNRs of the flash responses in each class shown.

The mechanisms by which the background photons added noise and decreased the SNRs were different in different cell types. In the rod and horizontal cell the mean flash response amplitude, the signal for our purposes, was reduced to 40-508 of its dark-adapted value upon background illumination, while the amplitude of the random, low-frequency fluctuations of membrane potential, the noise, was markedly increased. The high-frequency membrane noise was little afferted in these cells.

In the spiking cell, the background illumination produced a steady depolarization (not shown in the Figure, see legend) and a maintained spike activity, plus a noticeable increase in lowfrequency noise and an enormous increase in high-frequency noise. one component of this high-frequency noise appears as spikes with amplitudes of a few millivolts (arrows). It should be noted that, although background illumination of ten produces an initial increase in maintained spike activity, the main part of this increase is usually of temporary nature (see below).

Quantitative aspects of membrane noise in horizontal cells.

All three recordings in Fig. 1 gave the impression that the frequency range of the background-induced fluctuations was similar to that of the flash response. Only for three horizontal cells do we have long enough recordings sufficiently free from electrode noise to test the above impression rigorously. Fig. $2 \mathrm{a}$ shows $10 \mathrm{~s}$ segments of membrane potential recordings from one of these cells. The top records are taken in darkness and the lower in light $\left(0.58 \mathrm{Rh} * \mathrm{~s}^{-1}\right)$.

The power spectra of the fluctuations were compared using an FFT algorithm and are shown in Fig. 2b. Both the dark and background spectra can be divided into a high $(>2 \mathrm{~Hz})$ and a low $(<1 \mathrm{~Hz})$ frequency component. The high frequency components superimpose sufficiently to suggest that they are largely independent of light. The variance, $\sigma^{2}$, of the low frequency component, however, increased from $0.0095 \mathrm{mv}^{2}$ in darkness to $0.038 \mathrm{mv}^{2}$ in 1 ight. 

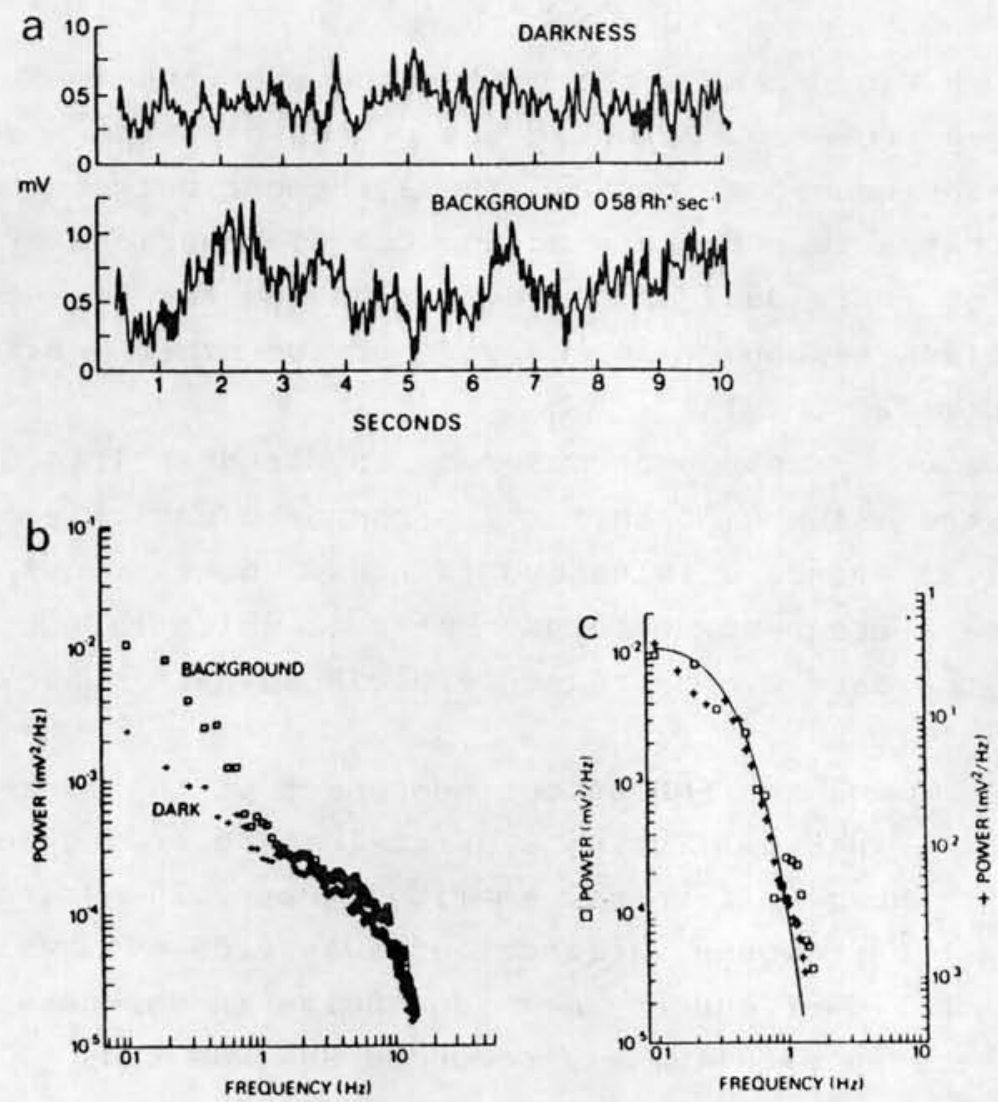

Fig. 2. Horizontal cell membrane fluctuations duiling darkness and under background illumination. (a). Ten second segments of membrane potential are shown during darkness (top trace) and background illumination (bottom trace). These digitized records were low pass filtered ( $<5 \mathrm{~Hz}, 24 \mathrm{~dB}$ per octave) and digitized at sampling intervals of $10 \mathrm{~ms}$. The resting membrane potential and flash sensitivity were $-41 \mathrm{mV}$ and $8.9 \mathrm{mV} / \mathrm{Rh}$, respectively, in darkness and $-46 \mathrm{mV}$ and $5.9 \mathrm{mV} / \mathrm{Rh}$ * during backgluund illumination. The background illumination was $500 \mathrm{~nm}$ light producing $0.58 \mathrm{Rh}^{\star} \mathrm{s}^{-1}$ and covered a cilcular area $1.5 \mathrm{~mm}$ in diametel centered on the impaled horizontal cell. (b). Powel spectra of membrane potentials. A 1024 pt Fast Foulier Transfolm Algoi i'hm was used to calculate the power spectral density of twenty $10-s$ segments from darkness and seventeen $10-$ s segments during background illumination. The average spectia are shown. Five point smoothing was applied to points above $1.2 \mathrm{~Hz}$. The variances of the low frequency components (calculated as the area under the power spectral density curve for frequencies $\leq 1.2 \mathrm{~Hz}$ ) were $\sigma^{2}$ background $=0.038 \mathrm{mV}^{2}$ and $\sigma^{2}$ dark $=0.0095 \mathrm{mv}^{2}$. ( $(\bar{c})$. Comparison of background-induced fluctuations and light responses. Squares plot the difference of the two spectra shown in (b). Pluses show the spectral density of 20-s segments which contained responses to dim flashes (0).24 Rh*/ flash). This is an average spectrum of four segments, diqitized at $19.5 \mathrm{~ms}$ intervals and low pass filtered at $10 \mathrm{~Hz}, 24 \mathrm{~dB} /$ octave. The ordinate for this light flash spectrum is shown on the right. The solid line is the spectral density of a model response fitted to the average light response during the background. The best model fit obtained used a "Poisson model" (13) having five stages with time constants of $195 \mathrm{~ms} / \mathrm{stage}$. 
The squares in Fig. $2 \mathrm{c}$ show the difference spectrum (backgrounddark) obtained from Fig. $2 \mathrm{~b}$ while the pluses plot the spectrum of small flash-induced responses. The agreement between these spectra indicates that background-induced fluctuations arise by a summation of individual photon events having the time course of the dim flash response. An analysis of two other horizontal cells gave similar results.

The low-frequency component observed in darkness (Fig. 2b) was analysed on the assumption that it is composed entirely of isomerization-like events originating in rods. These events were found to have a frequency of $0.037 \mathrm{Rh}^{*} \mathrm{D}^{-1}$, which is not very different from the dark event frequency $0.028 \mathrm{Rh}^{\star} \mathrm{DS}^{-1}$ observed in single rods (15).

We have determined the SNR of the lesponses of the dark-adapted horizontal cell in Fig. 1 using a drift-free recording encompassing 21 responses. The mean response amplitude was $2.26 \mathrm{mV}$ (range 1.84$2.85 \mathrm{mV}$ ) and the lesponse variance, $\sigma^{2}$, was $0.06 \mathrm{mv}^{2}$, while the variance of the low-frequency membrane noise in darkness was $0.0095 \mathrm{mv}^{2}$. The intracellularly recorded SNR was thus $2.26 / \sqrt{(0.06)+(0.0095)}=8.6$.

The result of this level two, physiological, SNR calculation (see Introduction) can be compared with the level one, maximum realizable SNR obtained for the same cell and stimulus: The receptive field was found to encompass 340 rods, and the integration time was $1.3 \mathrm{~s}$. Thus, applying Eq. (1), the upper limit SNR for the responses to $0.3 \mathrm{Rh}$ * $\mathrm{flashes}$ would be 9.5 (SNR = $0.3 \times 340 / \sqrt{(0.3 \times 340+(0.028 \times 1.3 \times 340)})$.

Against background, a level one calculation gives an SNR of 5.0 (the intensity of the background illumination was $0.92 \mathrm{Rh}^{\star} \mathrm{s}^{-1}$, and the integration time was then $1.0 \mathrm{~s})$. Due to large background noise the variance of the lesponse amplitudes cannot be accurately measured. However, by assuming that response standard deviation was reduced by the same factor as the mean response amplitude we could estimate the SNR of the intracellularly recorded responses against background to 3.5 (the variance of the low-frequency membrane noise under this background was $0.06 \mathrm{mv}^{2}$ ). 
The above calculations and the examples illustrated in Figs. 1 and 2 suggest that the rod noise originating from photoisomerizations and dark events are dominating factors limiting the SNR of the horizontal cells and hence, by implication, the signal transmission through the distal retina. Observe that this conclusion applies to retinae which are dark-adapted or exposed to very weak background illuminations.

\section{Extracellularly recorded increment thresholds}

A simple signal-to-noise consideration based on Eq. (1) requires that for responses with constant SNR the stimulus intensity at threshold should increase with the square root of background intensity (providing $E_{B} \gg E_{D}$ ) (31, 33). Barlow (11) has pointed out that, in psychophysical experiments, using suitable stimulus parameters, such a square root region can be detected. This square root dependency is seen in $\log -\log$ increment threshold curves between the "horizontal level" representing the dark-adapted thresholc and the $45^{\circ}$ part representing the Weber region where threshold increase is proportional to background increase.

We have measured increment threshold curves for 7 frog ganglion cells using extracellular recording and $0.11 \mathrm{~mm}$ stimulus spots against full field backgrounds. Just after turning on a new background, we often observed a radical change in maintained activity (see Fig. 1), and a reduced SNR at threshold. However, in the experiments referred to here the maintained activity was allowed to return to near its initial (low) level before we began the threshold determination. This was done after each new background presentation.

In all experiments the square root relation predicted by Eq. (1) was seen within a limited region up to background intensities of 50-250 $\mathrm{Rh} * \mathrm{~S}^{-1}$ and increment threshold intensities 3-10 times the dark-adapted threshold. Fig. 3 shows an experiment with an unusually expanded square root region between the dark-adapted level and the $45^{\circ}$ line representing the weber relation.

Experiments of the type shown in Fig. 3 seem to support the idea that the threshold responses result from an SNR type "determination" made by the retina and that simple level one calculations can accurately describe the SNRs for responses against weak background illuminations. However, a close analysis of these results reveals some complications. The square root law gives us 
a constant SNR for the physical stimulus-background relation seen through a constant time-window including a constant period of background flux. We know, however, that the integration time of the ganglion cell decreases monotonically with increasing photon flux (20). For example, at the upper end of the square root region in Fig. 3 the integration time was some 408 of the value at the lower end. Thus there is no simple physiological optimization connected with the precise square root relation.

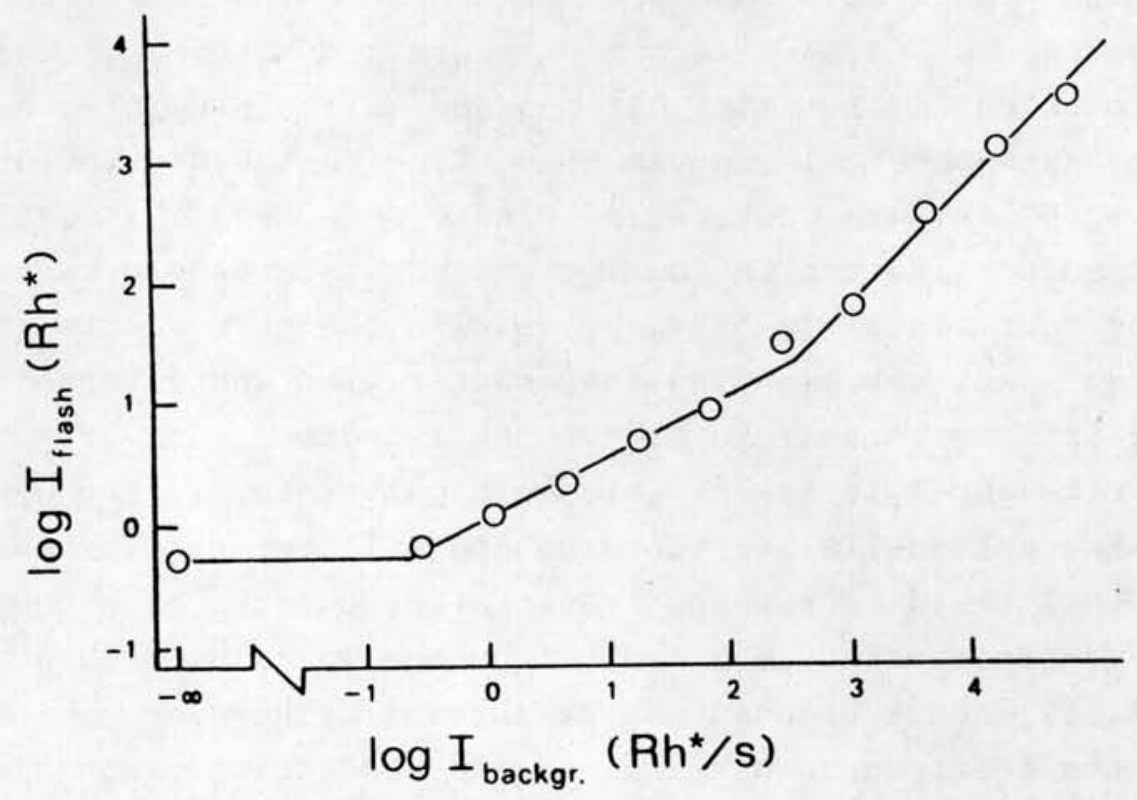

Fig. 3. Increment threshold function of Rana temporaria ganglion cell. Ordinate : log threshold intensity $\left(\mathrm{Rh}^{\star}\right)$ of flash stimulus with $0.1 .1 \mathrm{~mm}$ spot. Abscissa: $\log$ intensity $\left(\mathrm{Rh}^{*} \mathrm{~s}^{-1}\right)$ of full field background. The horizontal line represents the absolute threshold, while the middle and right (oblique) lines show square root and Weber relations, respectively. Temp. $11.5^{\circ} \mathrm{C}$. Cell type: class $1-2$.

Another objection concerns the SNR at threshold. A threshold intensity determines only a sensitivity level. Even when the maintained activity is approximately unchanged, we cannot be sure that the reliability (SNR) of the response at threshold remains constant when we change the background intensity. (The spontaneous firing of frog ganglion cells at $11.5^{\circ} \mathrm{C}$ is often less than 10 spikes per hour, implying that changes in maintained activity cannot always be easily detected). To accurately measure the SNR at threshold levels in extracellular recordings, we have 
to rely on frequency of response functions. These data can reveal whether the reliability remains constant during background illumination.

Frequency of response functions under dim background illumination

The recording of frequency of response functions was used here as one experimental way of estimating the amount of noise limiting the reliable detection of a stimulus by the ganglion cell. The particular hypothesis put to test was whether that noise could be wholly accounted for by the photon noise of the stimulus and background plus the dark noise from random photon-like events in the rods.

The experimental protocol was as follows: First, threshold intensities were determined for a small-spot flash, for a largespot flash, and for a small-spot ON-step, all centered on the receptive field of the cell. The integration time and receptive field size of this cell could be obtained from these protocols (see Methods). Then we presented a long series of small-spot flash stimuli in the threshold range of intensities, alternating between intensities above and below the estimated threshold. As most cells had a very low frequency of spontaneous discharges $(<0.5$ spikes/min), the occurrence of one or several spikes during a period $0.5-2.5 \mathrm{~s}$ after the flash was considered a response, and still "false" responses were rare. The response latency near threshold in these conditions was hardly ever less than $0.5 \mathrm{~s}$ and the integration time of the response was about $2 s(20)$. At each of 7-8 intensities, the relative response frequency was obtained from a total of 12 trials by taking the fraction of flashes that elicited a response in the $0.5-2.5 \mathrm{~s}$ time window.

In Fig. 4, the symbols give the results of three such frequency of response experiments against weak backgrounds. The data are to be compared with the curves, which express the theoretical prediction of the hypothesis: the curves give, as a function of mean flash intensity, the probability that the number $(x)$ of stimulus, background and dark events occurring in the cell's receptive field within its integration time shall exceed a certain threshold number (c) given by the observed 508 response level. As $x$ is poisson-distributed around a mean value, these probabilities $P(x \geq c)$ are cumulative Poisson probabilities (12). 


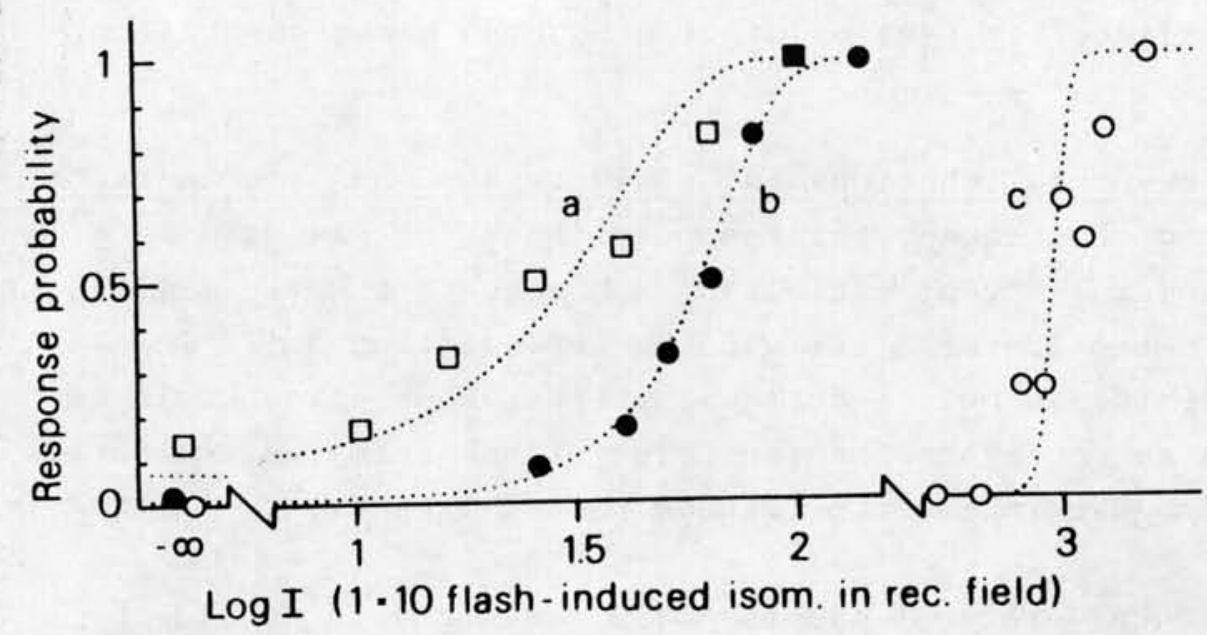

Fig. 4. Frequency of lesponse functions of three Rana ganglion cells $(a, b$ and $c)$ recorded under background illumination. Abscissa: $\log$ (mean) intensity of flash with $0.11 \mathrm{~mm}$ spot. Ordinate: fraction of flashes producing a spike response. The symbols showing response frequencies in darkness $(-\infty)$ give the probabilities of "false" responses calculated from the observed rates of spontaneous spikes. The dotted curves have been constructed as described in the Text. They represent frequency of response curves of idealized cells operating according to Eq. (1).

Background intensities: $0.46 \mathrm{Rh}^{\star} \mathrm{s}^{-1}(\mathrm{a}, \mathrm{b}), 18 \mathrm{Rh} \star^{-1}(\mathrm{c})$. Numbers of rods in receptive field: 1190 (a), 890 (b) and 600 (c). Temp. $11.5^{\circ} \mathrm{C}$. Cell classes: (a), class 3, (b), class 3, (c), class $1-2$.

The curves in Fig. 4. can be said to represent a level one SNR calculation, while the data points express recorded (level two) SNRs. If the data points are well fitted by a calculated frequency of response curve, then the SNR of the cell's reaction to a given stimulus corresponds to the SNR calculated for that stimulus intensity using Equation (1) and the parameters defining the curve.

Jf the data points indicate a flatter function than the curve, this means that threshold detection is affected by additional noise not included in the calculation producing the curve (12). For two of the experiments shown, the agreement between data and curve is reasonable; in the third experiment (c), the data points indicate a significantly flatter function, i.e. additional noise. our general conclusion from experiments of this type is that under dim backgrounds the ganglion cells are not far from operating as "perfect detectors" linearly integrating photon events from their receptive fields, and reliably producing a response whenever 
the event number exceeds a certain value.

\section{SNR-limiting noise in darkness}

The reasonable agreement between curves and data points in Fig. 4 suggests that if corresponding frequency of response experiments were carried out without background this would enable us to experimentally determine the level of spontaneous noise limiting the threshold in darkness. We could deduce the noise level in a quantitative way by formally assuming that all possible noise originates from photon-like events in rods. We could then compare this empirically determined noise with the frequency of dark rod events described by Baylor et al. (15).

In these experiments, frequency of response functions were recorded from fully dark-adapted cells without background, and theoretical frequency of response curves were fitted to the data (of the type shown in Fig. 4). The fit was optimized by finding the threshold and rate of dark noise giving maximum likelihood to the set of data. The data points showing response probability. in darkness ( Log $I=-\infty)$ were excluded and used in a separate calculation (see legend to Table 1).

Table 1 gives the results of six such experiments. It is clear that the dark event rates calculated from the frequency of response functions and from the rates of spontaneous spike activity show a large variation and generally are much higher than the dark event rate $0.006 \mathrm{Rh}{ }^{*} \mathrm{DS}^{-1}$ estimated for frog rods at $11.5 \mathrm{C}$ (see Methods and Biological Data).

This same conclusion applies to dark noise values calculated from thresholds in darkness and against weak backgrounds using the equation

$$
E_{S} / \sqrt{E_{S}+E_{D}}=2 E_{S} / \sqrt{2 E_{S}+E_{B}+E_{D B}}
$$

where $\mathrm{Z}$ is the factor by which the background-induced events $E_{B}$ have increased the intensity of the threshold stimulus $E_{S}$, and $E_{D B}$ the dark events summed during the "light" integration time, i.e. the shortening of $\underline{t}_{i}$ by background has been taken into account $(20)$.

The rationale behind this calculation is the assumption that the threshold response in darkness and against bickground represent the same SNR. For this calculation we thus used only experiments where the background did not appreciably affect the maintained spike activity. In five experiments the dark noise frequencies 
calculated by thismethod were $0.01,0.05,0.09,0.1$ and $0.75 " \mathrm{Rh} * \mathrm{D}^{-1} "$.

TABLE 1. Noise affecting threshold responses of dark-adapted Rana ganglion cells estimated by two independent procedures: (1) from frequency of response (FOR) curves as described in the Text, and (2) from spontaneous spike activity averaged over more than 1 hour. The latter method uses the assumed Poisson distribution of the number of noise events occurring within the receptive field (RF) within an integration time: the mean of that distribution is determined such that the probability for the event number to exceed the observed 508 flash threshold equals the observed probability of getting at least one spontaneous spike within one integration time.

\begin{tabular}{|c|c|c|c|c|c|}
\hline Cell No. & $\begin{array}{l}\text { Absolute } \\
\text { threshold } \\
\left.(\mathrm{Rh})^{*}\right)\end{array}$ & $\begin{array}{l}\text { Number of } \\
\text { rods in } \mathrm{RF}\end{array}$ & $\begin{array}{l}\text { Integration } \\
\text { time (s) }\end{array}$ & $\begin{array}{l}\text { Dark noise } \\
\text { from best- } \\
\text { fitting FOR- } \\
\text { curve" }\left(\mathrm{Rh}^{\star} \mathrm{D}^{-1}\right) "\end{array}$ & $\begin{array}{l}\text { Dark noise from } \\
\text { spontaneous acti- } \\
\text { vity " }\left(\mathrm{Rh}^{\star} \mathrm{D}^{\mathrm{s}}{ }^{-1}\right) "\end{array}$ \\
\hline 1 & 0.011 & 1190 & 0.8 & 0.090 & 0.196 \\
\hline 2 & 0.083 & 600 & 1.7 & 0.147 & 0.205 \\
\hline 3 & 0.012 & 1880 & 2.7 & 0.008 & 0.017 \\
\hline 4 & 0.056 & 890 & 1.7 & 0.344 & 0.273 \\
\hline 5 & 0.059 & 680 & 2.1 & 0.448 & 0.717 \\
\hline 6 & 0.160 & 530 & 2.4 & 0.083 & 0.542 \\
\hline
\end{tabular}

Thus all three methods for estimating the dark noise limiting the SNR of fully dark-adapted threshold response at $11.5^{\circ} \mathrm{C}$ indicate that, with a few exceptions, the events described by Baylor et al. (15) can constitute only a small fraction of this noise. Observe that we do not maintain that the dominating extra noise is composed of isomerization-like events. We just "translate" it to that form and formally express the noise power as a rate of equivalent photon-like events.

A possible source of additional noise.

When searching for a possible source of additional noise limiting the dark-adapted thresholds at $11.5^{\circ} \mathrm{C}$ we have noticed that large stimulus fields, which include a significant part of the inhibitory receptive field surrounding the excitatory field (8), produce flatter frequency of response curves than the small stimulus spots (diam. $0.11 \mathrm{~mm}$ ) used in the experiments described above. Fig. 5 shows an example of this; two frequency of response curves have been recorded for the same cell against a weak background, one with a small (filled circles; same as cell "b" in Fig. 4) 
and the other with a large (open squares) stimulus spot. The curve represents a level one calculation. The data obtained with a small stimulus spot are well fitted by this calculation based on photon noise in background and stimulus, while the data obtained with the large stimulus seem to be affected by additional noise.

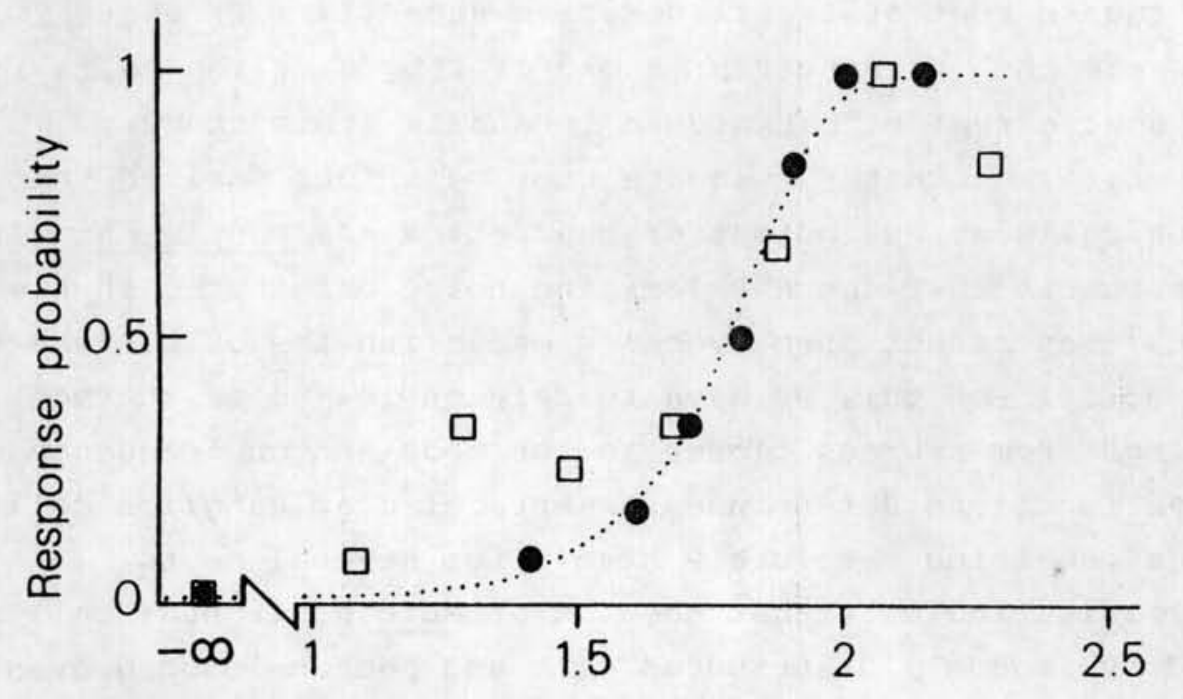

$\log I \quad(1=10 \mathrm{flash}$-induced isom. in rec. field)

Fig. 5. Frequency of response functions recorded from one cell with two different-sized stimulus spots: a small one $10.11 \mathrm{~mm}$, filled circles) and a large one $(0.8 \mathrm{~mm}$, squares). Same cell and conditions as in Fig. $4 \mathrm{~b}$; the circles and the theoretical curve are the same as shown there. The deviation of the squares from these indicatesextra noise activated by the large spot. Coordinate axes as in Fig. 4.

\section{DISCUSSION}

Several lines of evidence support the hypothesis that random isomerizations (photon and spontaneous) in rods limit threshold detection by the dark-adapted retina and the visual system. The SNR of dark-adapted depolarizing bipolar cells in the dogfish retina is limited by photon-like noise apparently originating from the rods (4), and the estimated flux of "dark light" postulated to limic the absolute chreshold of human vision $(1,9,11)$ is comparable to the rate of spontaneous events observed in darkadapeed monkey rods $(16)$. Analysing the signal tiansmission chrough the dark-adapted Bufo marinus rezina $\left(a=20^{\circ} \mathrm{C}\right)$ Copenhagen and Reucer (19) have concluded shat the dark events observed in coad rods (15) seem to form an important noise source limiting 
the SNR of the most reliable channels.

Further, amphibian ganglion cells have been seen to obey two square root laws which are consistent with the simple signal-tonoise considerations expressed by Eq. (1): 1) during signal transmission through the toad retina the SNR of the neurons increases as the square root of their summative area (19); 2) there is a range where the increment threshold of frog ganglion cells increases as the square root of background intensity (this study).

However, although these square root relations derived from the ganglion cells at the output of the retina are consistent with the hypothesis that the SNR-limiting noise originates in the receptors, they cannot unequivocally establish the origin and nature of the noise. For this we have to rely on low-noise intracellular recordings from neurons closer to the rods and on frequency of response functions determined in extracellular ganglion cell recordings remaining absolutely stable for several hours.

Our results indicate that the SNR of Bufo horizontal cell is limited by randomly distributed dark and photon-induced events. It is further obvious that the randomly distributed photons in a weak background illumination may play a key role in limiting the reliability (SNR) of ganglion cell responses. In complete darkness, spontaneous rod events seem to limit the SNR of many reliable Bufo ganglion cells at $20^{\circ} \mathrm{C}$ (19) and probably - even at $11.5{ }^{\circ} \mathrm{C}$ - of occasional dark-adapted Rana ganglion cells (see cell no. 3 in Table 1 of the present study). However, in most Rana ganglion cells at this low temperature, it appears that there is an additional noise source more prominent than the dark events in the rods.

If the recorded noise is only somewhat higher than the calculated "level one" value we have no reason to assume that the extra noise originates postsynaptically to the receptors. Our level one calculations do not take into account the amplitude variation of the receptor events as seen by the ganglion cell. First, the amplitudes of the individual dark and background-induced events show a considerable variation (15). Secondly, the amplitude of the isomerization signal reaching the ganglion cell will depend on the isomerization site in the receptive field. In both cases, the amplitude variation would add a multiplicative noise $(30)$.

However, even considering the multiplicative noise aspect, the 
rate of dark events is so $10 \mathrm{w}$ at $11.5^{\circ} \mathrm{C}$ that it only rarely can form the dominating factor limiting the reliability of dark-adapted ganglion cell responses. Experiments with small and large stimulus spots indicate that the extra noise grows when we include larger parts of the inhibitory zone surrounding the excitatory receptive field. This finding implies that neurons having extensive lateral interactions can introduce noise that impinges on the ganglion cell. One must therefore implicate neurons postsynaptic to the receptors as the cause of this noise.

The receptive field of a frog ganglion cell has a complicated structure of concentric excitatory and inhibitory zones 17,8 , $21,26)$. This organization is not related to a simple signal detection of the type discussed here, but to more complex aspects of analysis such as motion detection and contrast sensitivity (6). It is thus understandable that a stimulus activating large regions of the inhibitory surround evokes antagonistic interactions in the inner plexiform layer, and that these interactions will degrade the ganglion cell's reliability for the simple flash detection studied here.

As the inhibitory "surround" overlaps the excitatory centre (5) it is probable that such interactions always introduce some noise. They are at least partly mediated by amacrine cells which may produce the background-induced increase in high-frequency membrane noise seen in Fig. 1.

Such retinal noise of proximal origin has been postulated by many investigators, among them Frishman and Levine (25) who studied the maintained discharge of cat retinal ganglion cells.

\section{ACKNOWLEDGEMENTS}

We are grateful for the discussions and valuable insights provided by Drs. Jonathan Ashmore, Simo Hemilä and Jack Belgum. We thank Maj-Len Grönholm for many kinds of technical assistance, and Dr. Karl Johan Donner for the curve-fitting computer programs. This study was supported by NIH EY 01869 (DRC) and by the National Research Council for Sciences in Finland (TR). Dr. Reuter was a recipient of an ASLA-Fulbright travel and research grant and a Research to Prevent Blindness Travel Award. Dr. Copenhagen was the recipient of a John S. Adams Scholars Award from Research to Prevent Blindness. 


\section{REFERENCES}

1. Aguilar M, Stiles WS (1954) Optica Acta 1:59-65

2. Ashmore JF, Copenhagen DR (1983) J Physiol 340:569-597

3. Ashmore JF, Falk G (1977) Nature, London 270:69-71

4. Ashmore JF, Falk G (1980) J Physiol 300:115-150

5. Bäckström A-C (1981) Medical Biol 59:234-246

6. Bäckström A-C, Hemilä S, Reuter T (1978) Medical Biol 56:72-83

7. Bäckström A-C, Reuter T (1975) J Physiol 246:79-107

8. Barlow HB (1953) J Physiol 119:69-88

9. Barlow HB (1956) J opt Soc Am 46:634-639

10. Barlow HB (1964) In: Gese AC (ed) Photophysiology, vol. 2, Academic Press, New York, pp 163-202

11. Barlow HB (1965) Cold Spring Harbor Symp Quant Biol 30:539-546

12. Barlow HB (1977) In: Barlow HB, Fatt P (eds) Vertebrate Photoleception. Academic Preșs, London pp 337-351

13. Baylor DA, Hodgkin AL, Lamb TD (1974) J Physiol 242:685-727

14. Baylor DA, Lamb TD, Yau K-W (1979) J Physiol 288:613-634

15. Baylor DA, Matthews G, Yau K-W (1980) J Physiol 309:591-621

16. Baylor DA, Nunn BJ, Schnapf JL (1984) J Physiol 357:575-607

17. Copenhagen DR, Owen WG (1976) J Physiol 259:251-282

18. Copenhagen DR, Owen WG (1980) J Physiol 308:159-184

19. Copenhagen DR, Reuter $T$ (1986) Signal detection and dark noise in the retina of the toad (Bufo marinus). In preparation

20. Donner $K$ (1985) The ganglion cells of the frog retina: receptive field mechanisms and adaptational changes in these.

$\mathrm{Ph}$ D Thesis, University of Helsinki

21. Donner K, Grönholm M-L (1984) Vision Res 24:1807-1819

22. Donner KO, Reuter $T$ (1968) J Physiol 199:59-87

23. Fain GL (1975) Science, New York 187:838-841

24. Fain GL (1976) J Physiol 261:71-101

25. Frishman LJ, Levine MW (1983) J Physiol 339:475-494

26. Grönholm M-L, Reuter T (1981) Vision Res 21:1649-1652

27. Gyllenberg G, Reuter $T$, Sippel H (1974) Vision Res 14:1349-1357

28. Larsen LO, Pedersen JN (1982) Amphibia-Reptilia 2:321-327

29. Leeper HF, Nolmann RA, Copenhagen DR (1978) Nature, London $275: 234-236$

30. Lillywhite PG (1981) Vision Res 21:291-296

31. Rose A (1942) Proc Inst Radio Engrs 30:293-300

32. Schwartz EA (1975) J Physiol 246:617-638

33. de Vries H (1943) Physica 10:553-564 